\title{
Neurobiological substrates of major psychiatry disorders: transdiagnostic associations between white matter abnormalities, neuregulin 1 and clinical manifestation
}

\author{
Jia Duan, MD; Yange Wei, MD; Fay Y. Womer, MD; Xizhe Zhang, PhD; Miao Chang, MD; \\ Yue Zhu, MD; Zhuang Liu, PhD; Chao Li, MD; Zhiyang Yin, MD; Ran Zhang, MD; \\ Jiaze Sun, MD; Pengshuo Wang, MD; Shuai Wang, MD; Xiaowei Jiang, MD; \\ Shengnan Wei, MD; Yanbo Zhang, MD, PhD; Yanqing Tang, MD, PhD; Fei Wang, MD, PhD
}

\begin{abstract}
Background: Schizophrenia, bipolar disorder and major depressive disorder are increasingly being conceptualized as a transdiagnostic continuum. Disruption of white matter is a common alteration in these psychiatric disorders, but the molecular mechanisms underlying the disruption remain unclear. Neuregulin 1 (NRG1) is genetically linked with susceptibility to schizophrenia, bipolar disorder and major depressive disorder, and it is also related to white matter. Methods: Using a transdiagnostic approach, we aimed to identify white matter differences associated with NRG1 and their relationship to transdiagnostic symptoms and cognitive function. We examined the white matter of 1051 participants (318 healthy controls and 733 patients with major psychiatric disorders: 254 with schizophrenia, 212 with bipolar disorder and 267 with major depressive disorder) who underwent diffusion tensor imaging. We measured the plasma NRG1- $\beta 1$ levels of 331 participants. We also evaluated clinical symptoms and cognitive function. Results: In the patient group, abnormal white matter was negatively associated with NRG1- $\beta 1$ levels in the genu of the corpus callosum, right uncinate fasciculus, bilateral inferior fronto-occipital fasciculus, right external capsule, fornix, right optic tract, left straight gyrus white matter and left olfactory radiation. These NRG1-associated white matter abnormalities were also associated with depression and anxiety symptoms and executive function in patients with a major psychiatric disorder. Furthermore, across the 3 disorders we observed analogous alterations in white matter, NRG1- $\beta 1$ levels and clinical manifestations. Limitations: Medication status, the wide age range and our cross-sectional findings were limitations of this study. Conclusion: This study is the first to provide evidence for an association between NRG1, white matter abnormalities, clinical symptoms and cognition in a transdiagnostic psychiatric cohort. These findings provide further support for an understanding of the molecular mechanisms that underlie the neuroimaging substrates of major psychiatric disorders and their clinical implications.
\end{abstract}

\section{Introduction}

Schizophrenia, bipolar disorder and major depressive disorder have been separate diagnoses since the time of Kraepelin in the 1920s. ${ }^{1}$ However, diagnostic categories based on presenting signs and symptoms in psychiatric disorders may not capture the fundamental underlying mechanisms of dysfunction. ${ }^{2,3}$ Increasing evidence suggests that these 3 conditions overlap in terms of risk genes, ${ }^{4}$ endocrine and metabolic markers, ${ }^{5}$ brain structure and function, ${ }^{6,7}$ clinical symptoms ${ }^{8}$ and cognitive deficits. ${ }^{9}$ As a result, there has been increasing discussion in the psychiatric community about conceptualizing schizophrenia, bipolar disorder and major depressive disorder as major psychiatric disorders, a transdiagnostic continuum of disorders with common clinical characteristics. ${ }^{10}$ Such a reconceptualization allows us to analyze the biological features of these diagnoses at multiple levels ${ }^{11}$ and to explore symptom-specific/diseasegeneral biological mechanisms.

White matter alterations are a common neurobiological abnormality in major psychiatric disorders. ${ }^{7}$ Disruption of the white matter microstructure, detectable with diffusion

Correspondence to: F. Wang and Y. Tang, Department of Psychiatry, First Affiliated Hospital, China Medical University, 155 Nanjing North Street, Shenyang 110001, Liaoning, PR China; fei.wang@yale.edu; yanqingtang@163.com

Submitted Aug. 22, 2020; Revised Feb. 8, 2021; Revised Apr. 18, 2021; Accepted May 30, 2021

Cite as: J Psychiatry Neurosci 2021 September 1;46(5). doi: 10.1503/jpn.200166 
tensor imaging (DTI), can reflect compromises of myelin, the cytoskeletal structure or axonal density. Fractional anisotropy (FA) is a commonly used DTI metric for indexing white matter that can reflect many of the structural properties of white matter, such as axon density and degree of myelination. ${ }^{12,13}$ Studies using DTI have found shared white matter abnormalities in schizophrenia, bipolar disorder and major depressive disorder. ${ }^{14,15}$ Abnormal white matter is also related to clinical symptoms and cognitive function. ${ }^{16,17}$ Despite such evidence, the molecular mechanisms underlying white matter alterations remain unclear, and few studies have taken a transdiagnostic approach to understanding these mechanisms.

The neural growth factor neuregulin 1 (NRG1) is a likely candidate for the molecular basis of white matter alteration in major psychiatric disorders. NRG1 is a common susceptibility gene for schizophrenia, bipolar disorder and major depressive disorder, ${ }^{18-21}$ indicating that NRG1 is involved in the pathogenesis of major psychiatric disorders. Increasing genetic evidence and animal models have also shown altered NRG1-ErbB signalling in the nervous system in the presence of major psychiatric disorders. ${ }^{22-26}$ Furthermore, findings from recent animal and human studies suggest that NRG1 could affect white matter. ${ }^{27-33}$ Animal studies have shown that NRG1 plays an important role in myelination and influences the development of white matter. ${ }^{28,29}$ Human studies have found that NRG1 preferentially concentrates in the white matter; ${ }^{30}$ genetic variation in NRG1 is associated with white matter abnormalities (FA reduction) in psychiatric disorders. ${ }^{31-33}$ Nevertheless, it is not clear whether there are transdiagnostic white matter alterations associated with NRG1 in psychiatric disorders; as well, the effect of NRG1associated white matter abnormalities on common symptomatology and cognition is not well understood.

Our aim was to identify common transdiagnostic neuroimaging substrates of major psychiatric disorders with potential molecular mechanisms, and to clarify the clinical implications of those substrates. We assessed a large sample of patients with schizophrenia, bipolar disorder or major depressive disorder collected at a single site to characterize the relationship between plasma profile, brain structure and psychopathology. Specifically, we looked at plasma levels of NRG1- $\beta 1$ (one of the isoforms of NRG1, which has the ability to pass the blood-brain barrier and has been used in many studies ${ }^{34,35}$ ), white matter assessed via DTI, and clinical symptoms and cognitive function. We hypothesized that abnormal white matter in major psychiatric disorders would be associated with NRG1 plasma levels, and that such NRG1-associated white matter abnormalities would be correlated with clinical symptoms and cognitive function in a transdiagnostic fashion.

\section{Methods}

\section{Participants}

The study was conducted at a single site and included 1061 participants aged 13 to 55 years (260 patients with schizophrenia, 213 with bipolar disorder, 269 with major depressive disorder and 319 healthy controls). All participants with schizophrenia, bipolar disorder or major depressive disorder were recruited from the inpatient and outpatient services at Shenyang Mental Health Centre and the Department of Psychiatry, First Affiliated Hospital of China Medical University, Shenyang, China. Healthy controls were recruited from the local community by advertisement. All participants were Chinese. All procedures involving human participants were in accordance with the ethical standards of the institutional and/or national research committee, and with the 1964 Helsinki Declaration and its later amendments or comparable ethical standards. The study was approved by the Medical Science Research Ethics Committee of the First Affiliated Hospital of China Medical University (approval reference number [2012]25-1) and was consistent with the principles outlined in an internationally recognized standard for the ethical conduct of human research. After the study was thoroughly described to them, participants provided written informed consent (or their parents/guardians did, if they were younger than 18 years old).

All participants were evaluated by 2 trained psychiatrists to determine the presence or absence of Axis I psychiatric diagnoses using the Structured Clinical Interview for DSMIV Axis I Disorders (SCID) ${ }^{36}$ in those 18 years and older, and the Schedule for Affective Disorders and Schizophrenia for School-Age Children-Present and Lifetime Version (K-SADS$\mathrm{PL})^{37}$ in those younger than 18 years. To qualify for the study, patients met DSM-IV diagnostic criteria for schizophrenia, bipolar disorder or major depressive disorder but did not meet the criteria for any other Axis I disorder. Healthy controls did not have a current or lifetime Axis I disorder or a history of psychotic, mood or other Axis I disorders in first-degree relatives, as determined by a detailed family history. Participants were excluded if any of the following were present: current or past substance or alcohol abuse or dependence, or a concomitant major medical disorder; any MRI contraindications; or a history of head trauma with loss of consciousness for 5 minutes or longer, or any neurologic disorder.

For all participants, we obtained symptom measures using the 17-item Hamilton Depression Rating Scale, the Hamilton Anxiety Rating Scale, the Young Mania Rating Scale and the Brief Psychiatric Rating Scale. We evaluated cognitive function (executive function) using the Wisconsin Card Sorting Test.

\section{MRI acquisition}

All participants underwent DTI. We acquired MRI data using a GE Signa HDx 3.0 T scanner with a standard 8-channel head coil at the First Affiliated Hospital of China Medical University. We acquired DTI using a single-shot spin echo planar imaging sequence with the following parameters: repetition time $17000 \mathrm{~ms}$, echo time $85.4 \mathrm{~ms}$, image matrix = $120 \times 120$, field of view $=240 \times 240 \mathrm{~mm}^{2}, 65$ contiguous slices of $2 \mathrm{~mm}$ without a gap, 25 noncollinear directions $(\mathrm{b}=$ $1000 \mathrm{~s} / \mathrm{mm}^{2}$ ) together with an axial acquisition without diffusion weighting $(b=0)$, and voxel size $2.0 \mathrm{~mm}^{3}$. 


\section{Data preprocessing}

We processed DTI data using Pipeline for Analyzing braiN Diffusion imAges (PANDA; www.nitrc.org/projects/ panda), a fully automated program for processing brain diffusion images. We used default program parameters to process the images. Steps were as follows: (1) converting Digital Imaging and Communications in Medicine (DICOM) files into Neuroimaging Informatics Technology Initiative (NIfTI) images, (2) brain extraction, (3) correction for eddy-current distortion and head motion, (4) correction for b-matrix, and (5) computation for diffusion tensor metrics. Next, we performed diagonalization to yield 3 pairs of eigenvalues and eigenvectors. We calculated an index corresponding to relative motion by averaging the displacement relative to the first time point. We observed no significant differences in mean relative head motion parameters between groups (major psychiatric disorders $0.49 \pm 0.18 \mathrm{~mm}$ v. controls $0.47 \pm 0.13 \mathrm{~mm}, p=0.07)$. We also used head motion as a covariate in between-group analyses of FA values to explore the effect of head motion on DTI, and the results were consistent with our findings (Appendix 1, available at jpn.ca).

Based on the 3 eigenvalues, we computed FA on a voxelby-voxel basis. Then, PANDA nonlinearly registered individual FA images of native space to the FMRIB58_FA template in Montreal Neurological Institute space with a customized spatial resolution $(2 \times 2 \times 2 \mathrm{~mm})$. The normalized FA was overlaid with image edges derived from the FA template, and then snapshot pictures were produced for quality control of FA normalization. ${ }^{38}$ Ten participants (6 with schizophrenia, 1 with bipolar disorder, 2 with major depressive disorder and 1 healthy control) were excluded from subsequent analyses because of suboptimal imaging data quality (ghost, inadequate coverage of the brain or excessive head motion). Then, the FA images were smoothed with a $6 \mathrm{~mm}$ Gaussian kernel. We chose FA because it can reflect the connectivity of white matter, and a reduction in FA can reflect a decrease in myelination. ${ }^{12}$ Furthermore, previous studies have shown that variations in the NRG1 gene are associated with FA reduction in psychiatric disorders. ${ }^{31-33}$

\section{Measurement of plasma NRG1-ß1 levels}

We obtained blood samples from 331 participants (141 healthy controls and 190 patients with major psychiatric disorders) between $1000 \mathrm{~h}$ and $1500 \mathrm{~h}$. The $5 \mathrm{~mL}$ venous blood samples were collected and centrifuged at $2000 \mathrm{rpm}$ for 10 minutes, and then the plasma was stored at $-80^{\circ} \mathrm{C}$ for NRG1- $\beta 1$ measurement. We made plasma NRG1- $\beta 1$ determinations using the Human Premixed Multi-Analyte Kit (R\&D Systems, Inc.) with the Human Magnetic Luminex Assay (Bio-Rad Laboratories, Inc.). Samples were magnetically labelled using a human magnetic premixed microparticle cocktail of antibodies (lot number L120614). After participants provided blood samples, they underwent MRI within 24 hours.

\section{Statistical analysis}

We combined findings for patients with schizophrenia, bipolar disorder and major depressive disorder into a "major psychiatric disorders" group and carried out analyses to compare that group with the healthy controls. We examined between-group effects on demographic and clinical characteristics, NRG1- $\beta 1$ levels and cognitive measures using 2-sample $t$ tests or $\chi^{2}$ tests. Results were considered significant at $p<0.05$.

First, we performed between-group analyses of FA values in SPM12 (a MATLAB toolbox, MATLAB version 9.1; www. fil.ion.ucl.ac.uk/spm/m), with diagnostic group as an independent factor and age and sex as covariates. To maintain a balance between type I and type II errors, we thought carefully about the issue of statistical thresholding and decided that the optimal, most balanced approach would be to report results at voxel-wise $p<0.005$ with Gaussian random field (GRF) correction for cluster-level inference of $p<0.05$. Other studies have used the same threshold. ${ }^{39,40}$ We also performed exploratory analyses to investigate group differences in FA values with a stringent threshold (Appendix 1).

To further support the transdiagnostic approach, we performed analyses to compare the differences in plasma NRG1- $\beta 1$ levels and FA maps among patients with schizophrenia, bipolar disorder and major depressive disorder.

Second, using the group difference FA map as a mask, we performed voxel-based correlation analyses within the major psychiatric disorders group to examine relationships between FA values in the regions showing common white matter alterations and NRG1- $\beta 1$ levels, using age and sex as covariates. We used linear association for the correlation analyses, according to a linear correlation between NRG1 and the brain cortical tissue microarray from a previous study, ${ }^{41}$ and a further general linear model was applied to the association between NRG1 and white matter. ${ }^{41,42}$ Statistical significance was set at a voxel-level inference of $p<0.005$ with GRF correction for cluster-level inference of $p<0.05$. For clusters with a significant correlation between FA values and NRG1- $\beta 1$ levels in major psychiatric disorders, we extracted mean FA values for further analysis.

To further understand the clinical implications of NRG1associated white matter abnormalities, we performed partial correlation analyses (2-tailed), controlling for age and sex, in the major psychiatric disorders group to explore the relationships between the mean FA values of NRG1-associated white matter abnormalities, clinical symptoms and executive function. Results were considered significant at $p<0.05$, corrected for false discovery rate. Because the present sample had a wide age range (13-55 yr), we implemented 2-way analysis of variance with group (major psychiatric disorders or healthy controls) and age ( $\leq 30 \mathrm{yr}$ or $>30 \mathrm{yr})$ as betweensubjects factors, and sex as a covariate, to examine interactions between group and age. We also performed univariate analyses of variance to assess potential diagnosis $\times$ age interactions in extracted FA values and NRG1- $\beta 1$.

We also performed analyses to explore differences in individual diagnostic groups (schizophrenia, bipolar disorder, major depressive disorder and healthy controls) in terms of 
FA values, plasma NRG1- $\beta 1$ levels, symptoms and alterations in cognitive function (Appendix 1).

\section{Results}

\section{Demographic and clinical data}

Detailed participant demographic and clinical data are outlined in Table 1.

We found no significant differences in terms of age, sex or smoking status between patients with major psychiatric disorders and healthy controls. As expected, we found significant differences in scores on the Hamilton Depression Rating Scale, Hamilton Anxiety Rating Scale, Young Mania Rating Scale and Wisconsin Card Sorting Test $(p<0.001$, Table 1). We found no significant interaction between group and age in terms of FA values or NRG1- $\beta 1$ levels. Demographic, clinical and cognitive characteristics for patients with schizophrenia, bipolar disorder and major depressive disorder are listed in Appendix 1, Table S1. Demographic characteristics for participants who had NRG1- $\beta 1$ levels measured are listed in Appendix 1, Table S2.

\section{White matter across diagnostic groups}

Between-group analysis (major psychiatric disorders compared with healthy controls) showed that patients with major psychiatric disorders had significantly decreased FA values in several regions compared to healthy controls. These regions involved callosal, limbic-paralimbic-heteromodal, corticocortical and thalamocortical white matter, including the genu, body and splenium of the corpus callosum; the bilateral anterior and superior corona radiata; the bilateral posterior thalamic radiation; the bilateral anterior and posterior limb of the internal capsule; the bilateral external capsule; the bilateral superior longitudinal fasciculus; the cingulum; the bilateral inferior and superior fronto-occipital fasciculus; the bilateral cerebral peduncle; the fornix; the bilateral uncinate fasciculus; the right optic tract; the right mediodorsal thalamic nucleus; the left straight gyrus white matter; and the left olfactory radiation (Figure 1 and Appendix 1, Table S3).

Results showed common alterations of NRG1- $\beta 1$ levels and FA in people with schizophrenia, bipolar disorder and major depressive disorder compared to healthy controls, suggesting a transdiagnostic continuum of major psychiatric disorders (Appendix 1, Figure S5).

\section{NRG1-ß1 levels across diagnostic groups}

Compared to healthy controls, NRG1- $\beta 1$ levels were significantly lower in patients with major psychiatric disorders $(p=0.011$; Table 1$)$.

\section{Correlations between white matter alterations and NRG1- $\beta 1$ levels}

In the major psychiatric disorders group, 3 clusters showed a significant negative correlation between $\mathrm{FA}$ values and

Table 1: Participant demographics and clinical characteristics

\begin{tabular}{|c|c|c|c|c|}
\hline Variable $^{*}$ & $\begin{array}{l}\text { Healthy controls } \\
\qquad(n=318)\end{array}$ & $\begin{array}{l}\text { Major psychiatric } \\
\text { disorders } \\
(n=733)\end{array}$ & $t / \chi^{2}$ & $p$ value \\
\hline \multicolumn{5}{|l|}{ Demographics } \\
\hline Age at scan, yr & $28.59 \pm 10.19$ & $27.27 \pm 10.24$ & 1.93 & 0.054 \\
\hline Sex, M/F & $131 / 187$ & $267 / 466$ & 2.14 & 0.14 \\
\hline Smoker, yes/no/unknown & $27 / 132 / 159$ & $66 / 251 / 416$ & 0.99 & 0.32 \\
\hline \multicolumn{5}{|l|}{ Clinical characteristics } \\
\hline First episode, yes/no/unknown & NA & $475 / 202 / 56$ & - & - \\
\hline Medication, yes/no/unknown & NA & $484 / 247 / 2$ & - & - \\
\hline Duration, mo & NA & $33.79 \pm 56.26$ & - & - \\
\hline Hamilton Depression Rating Scale, total score & $1.26 \pm 2.29$ & $13.16 \pm 10.32$ & -28.006 & $<0.001$ \\
\hline Hamilton Anxiety Rating Scale, total score & $1.32 \pm 2.97$ & $11.14 \pm 10.15$ & -22.730 & $<0.001$ \\
\hline Young Mania Rating Scale, total score & $0.31 \pm 0.97$ & $3.40 \pm 6.85$ & -11.699 & $<0.001$ \\
\hline Brief Psychiatric Rating Scale, total score & $18.51 \pm 1.89$ & $29.08 \pm 9.91$ & -23.104 & $<0.001$ \\
\hline NRG1- $\beta 1 \dagger$ & $9.92 \pm 1.84$ & $9.48 \pm 1.97$ & 6.659 & 0.011 \\
\hline \multicolumn{5}{|l|}{ Wisconsin Card Sorting Test (cognitive function)‡ } \\
\hline Correct responses & $29.81 \pm 12.04$ & $24.00 \pm 11.84$ & 6.238 & $<0.001$ \\
\hline Categories completed & $3.83 \pm 2.17$ & $2.82 \pm 2.06$ & 6.134 & $<0.001$ \\
\hline Total errors & $18.25 \pm 12.14$ & $23.87 \pm 11.87$ & -6.001 & $<0.001$ \\
\hline Perseverative errors & $6.54 \pm 6.96$ & $9.58 \pm 9.16$ & -4.998 & $<0.001$ \\
\hline Nonperseverative errors & $11.59 \pm 6.98$ & $14.34 \pm 7.40$ & -4.825 & $<0.001$ \\
\hline \multicolumn{5}{|c|}{$\begin{array}{l}\mathrm{F}=\text { female; } \mathrm{M}=\text { male; } \mathrm{NA}=\text { not applicable; } \mathrm{NRG} 1=\text { neuregulin } 1 . \\
\text { *Data are presented as mean } \pm \text { standard deviation unless otherwise specified. } \\
\text { †Healthy controls, } n=141 ; \text { major psychiatric disorders, } n=190 . \\
\text { †Healthy controls, } n=249 ; \text { major psychiatric disorders, } n=470 .\end{array}$} \\
\hline
\end{tabular}




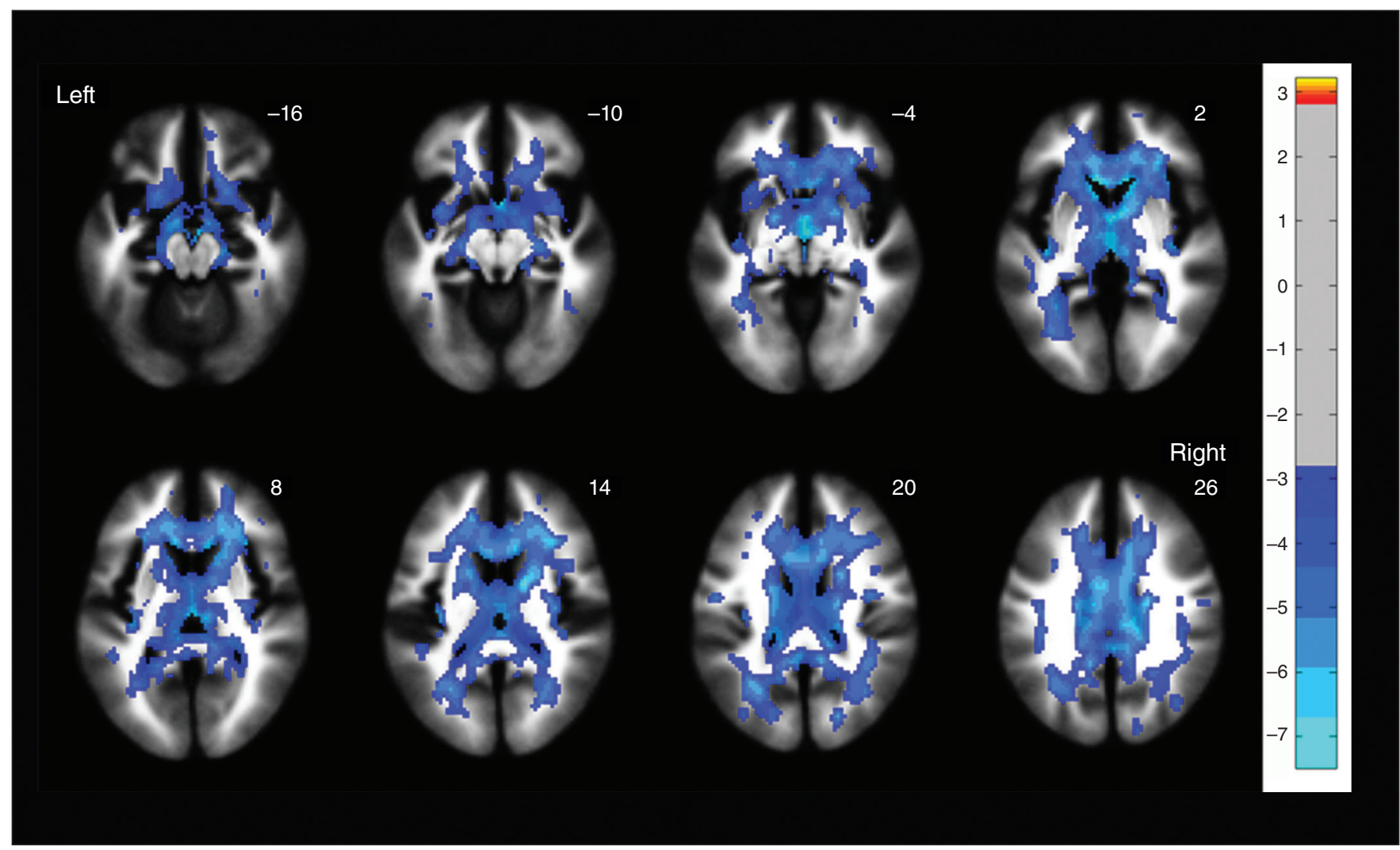

Figure 1: Significant differences in fractional anisotropy values between patients with major psychiatric disorders and healthy controls. Significance was set at $p<0.005$ at the voxel level, with Gaussian random field correction for cluster-level inference of $p<0.05$.

NRG1- $\beta 1$ levels. These clusters consisted of callosal, limbicparalimbic-heteromodal, corticocortical and thalamocortical white matter regions: white matter A included the genu of the corpus callosum, the right external capsule, the right uncinate fasciculus and the right inferior fronto-occipital fasciculus; white matter $\mathrm{B}$ included the fornix and the right optic tract; and white matter $C$ included the left right inferior fronto-occipital fasciculus, the left straight gyrus white matter and the left olfactory radiation (Figure 2 and Table 2).

\section{Correlations between white matter alterations and clinical variables}

In the major psychiatric disorders group, the somatic anxiety factor scores and the total score on the Hamilton Depression Rating Scale were positively correlated with FA values in all clusters that showed negative correlations with NRG1- $\beta 1$ (Table 3). Total score on the Hamilton Anxiety Rating Scale and categories completed on the Wisconsin Card Sorting Test were positively correlated with FA values in the white matter B cluster. We found no significant correlations between FA values in clusters that showed a negative correlation with NRG1- $\beta 1$ levels and the psychic anxiety, core depressive and anorexia factor scores on the Hamilton Depression Rating Scale, the Young Mania Rating Scale, the Brief Psychiatric Rating Scale or other scores on the Wisconsin Card Sorting Test.
Individual patient groups (schizophrenia, bipolar disorder or major depressive disorder) showed analogous white matter alterations, NRG1- $\beta 1$ levels and clinical manifestations (Appendix 1, Figures S1 to S5).

\section{Discussion}

This was the first study to use a transdiagnostic approach to identify abnormal white matter related to NRG1- $\beta 1$ levels and clinical and cognitive symptoms in major psychiatric disorders. We found widespread alterations in FA and decreased plasma NRG1- $\beta 1$ levels in the major psychiatric disorders group relative to healthy controls. Further analysis showed that FA values were related to NRG1- $\beta 1$ levels in the major psychiatric disorders group, predominantly in callosal, limbicparalimbic-heteromodal, corticocortical and thalamocortical white matter. Moreover, these NRG1-associated white matter abnormalities were related to depressive and anxiety symptoms and to executive function in the major psychiatric disorders group. We observed analogous alterations across the 3 disorders (schizophrenia, bipolar disorder and major depressive disorder) in terms of white matter alterations, NRG1- $\beta 1$ levels and clinical manifestations. These results add to an increasing body of literature implicating NRG1 in the mechanisms that underlie white matter abnormalities in psychiatric disorders and extend this literature by exploring the clinical implications of NRG1-associated white matter abnormalities. 


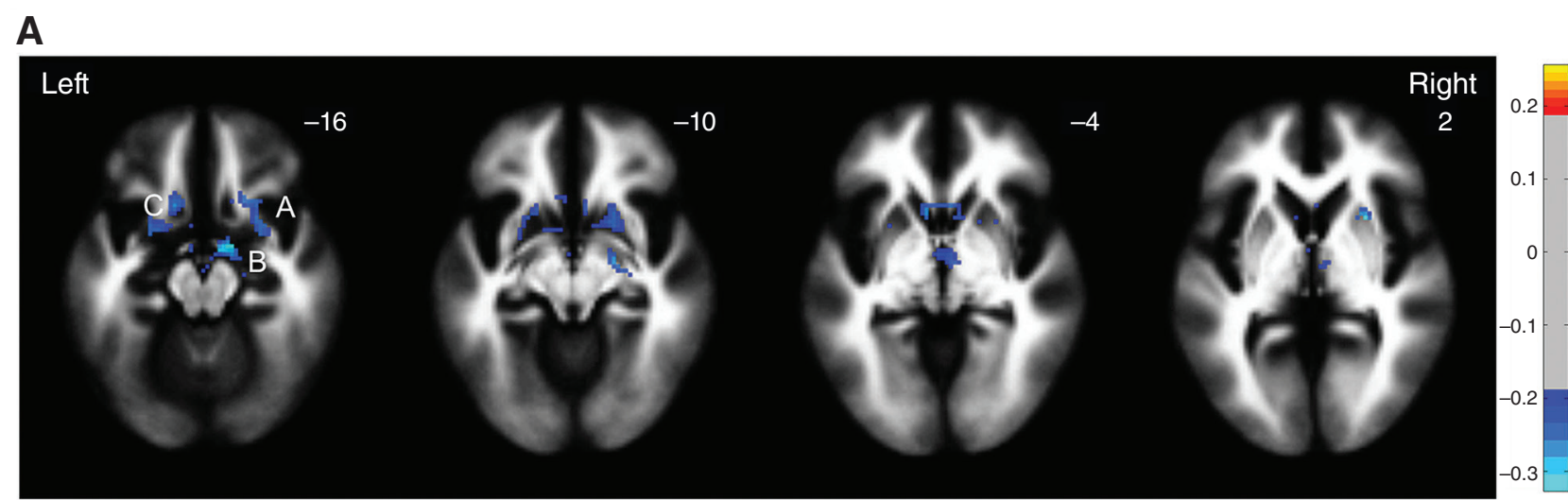

B
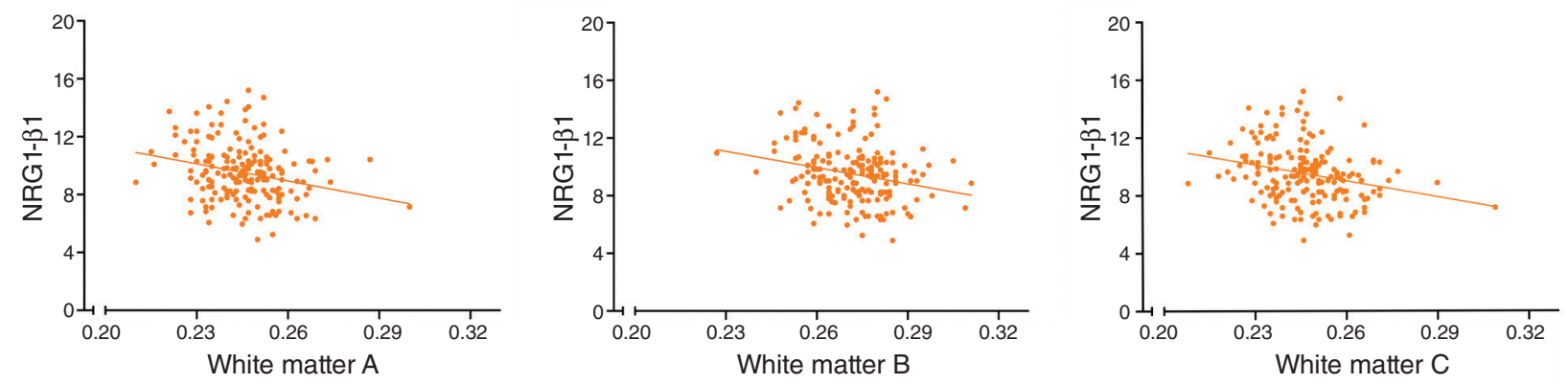

Figure 2: Patients with major psychiatric disorders showed significant correlations between fractional anisotropy in white matter and NRG1- $\beta 1$. (A) Regions that showed significant associations with NRG1- $\beta 1$ in patients with major psychiatric disorders. Significance was set at $p<0.005$ at the voxel level, with Gaussian random field correction for cluster-level inference of $p<0.05$. (B) Scatter plots of the association between fractional anisotropy and NRG1- $\beta 1$ in patients with major psychiatric disorders. White matter $A=$ genu of corpus callosum, right external capsule, right uncinate fasciculus, right inferior fronto-occipital fasciculus; white matter $B=$ fornix, right optic tract; white matter $C=$ left inferior fronto-occipital fasciculus, left straight gyrus white matter, left olfactory radiation.

Table 2: Patients with major psychiatric disorders who showed significant correlations between fractional anisotropy in white matter and NRG1- $\beta 1$

\begin{tabular}{|c|c|c|c|c|}
\hline Index & White matter label & $\begin{array}{l}\text { Cluster size, } \\
\text { voxels }\end{array}$ & MNI coordinates, $x, y, z$ & $R$ \\
\hline White matter A & $\begin{array}{l}\text { Genu of corpus callosum, right external capsule, right } \\
\text { uncinate fasciculus, right inferior fronto-occipital fasciculus }\end{array}$ & 283 & $26,12,4$ & -0.312 \\
\hline White matter B & Fornix, right optic tract & 183 & $14,-2,16$ & -0.307 \\
\hline White matter $\mathrm{C}$ & $\begin{array}{l}\text { Left inferior fronto-occipital fasciculus, left straight gyrus } \\
\text { white matter, left olfactory radiation }\end{array}$ & 161 & $-14,18,-18$ & -0.269 \\
\hline
\end{tabular}

$\mathrm{MNI}=$ Montreal Neurological Institute; NRG1 = neuregulin 1.

${ }^{*}$ Significance was set at $p<0.005$ at the voxel level, with Gaussian random field correction for cluster-level inference of $p<0.05$.

Associations between white matter alterations and NRG1-B1 levels in major psychiatric disorders

In this study, FA values ${ }^{14,43}$ and plasma NRG1- $\beta 1$ levels were decreased in the major psychiatric disorders group relative to healthy controls, consistent with the literature. ${ }^{44,45}$ In psychiatric disorders, NRG1 could have an effect on white matter alterations. Previous studies have demonstrated that NRG1ErbB signalling is associated with white matter at multiple levels in psychiatric disorders, from genetic variations to gene expression. Genetic imaging studies have found that variations in NRG1 are associated with white matter in the right uncinate fasciculus, corpus callosum, external capsule and inferior fronto-occipital fasciculus; ${ }^{35,42}$ the other NRG1associated white matter alterations reported in the present study have not been previously detected. Studies have also shown that the expression levels or function of NRG1 and ErbB are altered in major psychiatric disorders. ${ }^{46,47}$ Translational research suggests a causal role, because animal studies have found that decreased ErbB expression in white matter 
Table 3: Partial correlations between alterations in white matter integrity and clinical variables in patients with major psychiatric disorders

\begin{tabular}{|c|c|c|c|c|c|c|}
\hline \multirow[b]{2}{*}{ Clinical variable } & \multicolumn{2}{|c|}{ White matter $\mathrm{A}$} & \multicolumn{2}{|c|}{ White matter B } & \multicolumn{2}{|c|}{ White matter $\mathrm{C}$} \\
\hline & $r$ & $p$ value & $r$ & $p$ value & $r$ & $p$ value \\
\hline \multicolumn{7}{|l|}{ Hamilton Depression Rating Scale } \\
\hline Somatic anxiety (factor) score & 0.109 & $0.005^{\star}$ & 0.127 & $0.001^{*}$ & 0.102 & $0.009^{*}$ \\
\hline Psychic anxiety (factor) score & 0.066 & 0.10 & 0.04 & 0.31 & 0.078 & $0.048 \dagger$ \\
\hline Core depressive (factor) score & 0.059 & 0.13 & 0.053 & 0.17 & 0.073 & 0.06 \\
\hline Anorexia (factor) score & 0.063 & 0.11 & 0.074 & 0.06 & 0.065 & 0.10 \\
\hline Total score & 0.098 & $0.013^{*}$ & 0.097 & $0.013^{*}$ & 0.105 & $0.007^{\star}$ \\
\hline Hamilton Anxiety Rating Scale, total score & 0.09 & $0.027 \dagger$ & 0.1 & $0.013^{\star}$ & 0.078 & 0.054 \\
\hline Young Mania Rating Scale, total score & -0.038 & 0.35 & -0.047 & 0.25 & 0.019 & 0.63 \\
\hline \multicolumn{7}{|l|}{ Brief Psychiatric Rating Scale } \\
\hline Anxiety and depression (factor), score & 0.07 & 0.10 & 0.065 & 0.13 & 0.046 & 0.29 \\
\hline Lack of energy (factor), score & -0.025 & 0.56 & -0.094 & $0.028 \dagger$ & -0.068 & 0.11 \\
\hline Thought disorder (factor), score & -0.003 & 0.95 & -0.078 & 0.07 & -0.007 & 0.88 \\
\hline Activity (factor), score & 0.004 & 0.92 & -0.043 & 0.32 & 0.029 & 0.50 \\
\hline Hostility (factor), score & -0.003 & 0.95 & -0.058 & 0.18 & -0.002 & 0.97 \\
\hline Total score & 0.019 & 0.66 & -0.051 & 0.23 & -0.001 & 0.99 \\
\hline \multicolumn{7}{|l|}{ Wisconsin Card Sorting Test } \\
\hline Corrected responses & 0.074 & 0.11 & 0.064 & 0.16 & 0.071 & 0.12 \\
\hline Categories completed & 0.114 & $0.014 \dagger$ & 0.123 & $0.008^{*}$ & 0.11 & $0.017 \dagger$ \\
\hline Total errors & -0.061 & 0.19 & -0.062 & 0.18 & -0.066 & 0.16 \\
\hline Perseverative errors & -0.001 & 0.98 & -0.017 & 0.71 & -0.009 & 0.85 \\
\hline Nonperseverative errors & -0.105 & $0.023 \dagger$ & -0.073 & 0.12 & -0.103 & $0.026 \dagger$ \\
\hline
\end{tabular}

tracts, including the corpus callosum, could cause white matter alterations in major psychiatric disorders. ${ }^{48}$

It is possible that white matter alterations are influenced by NRG1 regulation of oligodendrocyte and myelination. Defects in NRG1-ErbB signalling could result in alterations of oligodendrocyte development and abnormal myelination, resulting in alterations of white matter. ${ }^{46,48}$ The potential pathophysiological role of NRG1 in psychiatric disorders might be to alter connections between the prefrontal cortex and other brain regions ${ }^{31}$ such as the genu of the corpus callosum, the uncinate fasciculus, the inferior fronto-occipital fasciculus and the external capsule. Several groups have assessed gene and protein expression of NRG1 and ErbB in postmortem brains and found dysregulations primarily in the prefrontal cortex in people with psychiatric disorders. ${ }^{28,49,50}$ There is also immunohistochemical evidence for impaired NRG1 signalling in the prefrontal cortex in people with psychiatric disorders. ${ }^{51}$ In addition, negative correlations between FA values and plasma NRG1- $\beta 1$ levels found in the major psychiatric disorders group in the present study might suggest compensatory effects under pathological conditions, playing an important role in brain plasticity. ${ }^{29,52}$ The negative correlation could be attributed to remyelination after white matter damage. As one of the few spontaneous repair processes in the central nervous system, remyelination can provide a certain degree of myelin reconstitution, but it remains insufficient for the deficit. ${ }^{53}$ White matter microstructure appears altered in major psychiatric disorders and may reflect decreased myelination compared to healthy controls. The relative lack of myelination may drive compensatory responses in people with major psychiatric disorders, resulting in the negative correlation between FA and expression levels of the myelinationrelated gene NRG1. ${ }^{4}$

\section{Associations between NRG1-associated white matter alterations, clinical symptoms and cognition in people with major psychiatric disorders}

In the present study, NRG1-associated white matter alterations were also associated with Wisconsin Card Sorting Test scores in patients with major psychiatric disorders. Previous studies have suggested a strong association between white matter alterations and cognition. ${ }^{55}$ In our study, executive function was correlated with FA values in the fornix and right optic tract in the major psychiatric disorders group. The fornix is part of the limbic system, and as the primary efferent network from the hippocampus, it is critical for cognitive function. ${ }^{56}$ Nestor and colleagues ${ }^{57}$ have reported reduced executive functioning correlated with fornix alteration in people with schizophrenia.

These NRG1-associated white matter abnormalities were also related to depressive and anxiety symptoms in our study. Reports have indicated that these NRG1-associated white matter abnormalities may be involved in emotion processing. ${ }^{58-60}$ The corpus callosum is critical to interhemispheric communication and to the integration of emotional 
information. ${ }^{58,59}$ Together, the uncinate fasciculus and inferior fronto-occipital fasciculus form the ventral external capsule, and these and the olfactory radiation contain pathways that extend into limbic brain regions that are important for emotional regulation. ${ }^{60,61}$ Interestingly, we observed that NRG1-associated white matter abnormalities were positively correlated with scores on the Hamilton Depression Rating Scale and the Hamilton Anxiety Rating Scale. The positive correlations we found might reflect the relationship between NRG1-associated white matter abnormalities and clinical symptoms (of depression and anxiety) under pathological conditions. Altered NRG1-associated white matter, which is more likely to be a patient trait-related change, ${ }^{62,63}$ might contribute to alterations in functional connectivity between prefrontal and limbic brain regions, ${ }^{64,65}$ and subsequently to dysfunction of emotional processing. ${ }^{66}$ However, FA differences may simply reflect changes in orientation-dependent aspects of white matter microstructure, because FA is not a direct measure of white matter integrity. Further interpretation of FA findings in relation to white matter integrity warrants further study. ${ }^{13}$

\section{Limitations}

This study had several limitations. First, most patients with a major psychiatric disorder were taking psychotropic medications at the time of the study, which may have altered white matter and NRG1- $\beta 1$ levels. However, previous studies have shown limited evidence for the effect of medications on white matter ${ }^{67}$ and NRG1- $\beta 1 .^{68}$ Further studies in medication-naive patients are needed to clarify these issues. Second, our sample had a relatively wide age range (13$55 \mathrm{yr})$, and the effects of age on white matter development may have confounded our findings. We performed an analysis to test for interactions between group and age and found no significant interaction between group and age on FA values and NRG1- $\beta 1$ levels. Regions that showed group $\times$ age interactions did not overlap with the regions in which NRG1-associated white matter abnormalities were located. Furthermore, we found no significant differences in age between the major psychiatric disorders group and the healthy controls. We also found no significant correlations between age and NRG1-associated white matter abnormalities in the major psychiatric disorders group. Further studies of white matter development could be performed to clarify the effects of age. Third, this study was cross-sectional, and we have limited details related to the longitudinal course and severity of symptoms. Clearly, longitudinal research is needed to better understand the relationships between white matter, NRG1- $\beta 1$ and clinical symptoms in psychiatric disorders. Fourth, it is not entirely clear to what extent plasma NRG1 levels reflect levels in the brain. However, even if NRG1 abnormalities in the blood were not a perfect mirror of pathological processes in brain, they could represent distinct molecular changes that are specific to the primary pathophysiology. ${ }^{69}$ As technology advances, in vivo levels of NRG1 in cerebrospinal fluid could be measured to verify results. In addition, smoking might be associated with
NRG1- $\beta 1$ levels. ${ }^{26}$ The effect of smoking on NRG1- $\beta 1$ in this study was unclear because of a lack of information on the association between the smoking status of patients with major psychiatric disorders and NRG1- $\beta 1$ data. There also might be complex associations between NRG1 and FA. Further studies are needed to clarify this issue.

\section{Conclusion}

This study used a dimensional approach to identify transdiagnostic white matter alterations that might represent important common neuroimaging substrates and indicate markers for potential biological mechanisms of major psychiatric disorders. The NRG1-associated white matter abnormalities found in our study could also be associated with depressive and/or anxiety symptoms and with executive function, and may represent a treatment target for psychiatry disorders.

Acknowledgements: The authors thank the members of the patient and healthy control groups for taking part in the study. The authors were supported by research grants from the National Science Fund for Distinguished Young Scholars (81725005 to FW), National Natural Science Foundation of China (81571311 to YT, 81571331 to FW), National Key Research and Development Program (2018YFC1311604 to YT, 2016YFC1306900 to YT, 2016YFC0904300 to FW), National High Tech Development Plan (863; 2015AA020513 to FW), Liaoning Science and Technology Project (2015225018 to YT), Liaoning Education Foundation (Pandeng Scholar to FW), Innovation Team Support Plan of Higher Education of Liaoning Province (LT2017007 to FW), Major Special Construction plan of China Medical University (3110117059 to FW).

Affiliations: From the Department of Psychiatry, First Affiliated Hospital of China Medical University, Shenyang, Liaoning, PR China (Duan, Zhu, Yin, R. Zhang, Sun, P. Wang, S. Wang, Tang, F. Wang); the Early Intervention Unit, Department of Psychiatry, Affiliated Nanjing Brain Hospital, Nanjing Medical University, Nanjing, PR China (Duan, Y. Wei, R. Zhang, F. Wang); the Department of Psychiatry and Behavioral Neuroscience, Saint Louis University, St. Louis, MO (Womer); the School of Biomedical Engineering and Informatics, Nanjing Medical University, Nanjing, Jiangsu, PR China (X. Zhang); the Department of Radiology, First Affiliated Hospital of China Medical University, Shenyang, Liaoning, PR China (Chang, Li, Jiang, S.Wei); the School of Public Health, China Medical University, Shenyang, Liaoning, PR China (Liu); the Department of Psychiatry, College of Medicine, University of Saskatchewan, SK (Y. Zhang).

\section{Competing interests: None declared.}

Contributors: Y. Tang and F. Wang designed the study. Y. Zhu, C. Li, Z. Yin, J. Sun, P. Wang, S. Wang, X. Jiang and S. Wei acquired the data, which J. Duan, Y. Wei, F. Womer, X. Zhang, M. Chang, Z. Liu, R. Zhang and Y. Zhang analyzed. J. Duan and M. Chang wrote the article, which Y. Wei, F. Womer, X. Zhang, Y. Zhu, Z. Liu, C. Li, Z. Yin, R. Zhang, J. Sun, P. Wang, S. Wang, X. Jiang, S. Wei, Y Zhang, Y. Tang and F. Wang reviewed. All authors approved the final version to be published and can certify that no other individuals not listed as authors have made substantial contributions to the paper.

Content licence: This is an Open Access article distributed in accordance with the terms of the Creative Commons Attribution (CC BY-NC-ND 4.0) licence, which permits use, distribution and reproduction in any medium, provided that the original publication is properly cited, the use is noncommercial (i.e., research or educational use), and no modifications or adaptations are made. See: https://creativecommons.org/licenses/by-nc-nd/4.0/ 


\section{References}

1. Kraepelin E. Manifestation of insanity. Z Gesamte Neurol Psychiatr $1920 ; 62: 1-29$

2. Cuthbert BN, Insel TR. Toward the future of psychiatric diagnosis: the seven pillars of RDoC. BMC Med 2013;11:126.

3. Ivleva EI, Clementz BA, Dutcher AM, et al. Brain structure biomarkers in the psychosis biotypes: findings from the bipolarschizophrenia network for intermediate phenotypes. Biol Psychiatry 2017;82:26-39.

4. Consortium C-DGotPG. Identification of risk loci with shared effects on five major psychiatric disorders: a genome-wide analysis. Lancet 2013;381:1371-9.

5. Goldsmith DR, Rapaport M, Miller B. A meta-analysis of blood cytokine network alterations in psychiatric patients: comparisons between schizophrenia, bipolar disorder and depression. Mol Psychiatry 2016;21:1696-1709.

6. Goodkind M, Eickhoff SB, Oathes DJ, et al. Identification of a common neurobiological substrate for mental illness. JAMA Psychiatry 2015;72:305-15.

7. Chang M, Womer FY, Edmiston EK, et al. Neurobiological commonalities and distinctions among three major psychiatric diagnostic categories: a structural MRI study. Schizophr Bull 2018; 44:65-74.

8. Lee RS, Hermens D, Naismith S, et al. Neuropsychological and functional outcomes in recent-onset major depression, bipolar disorder and schizophrenia-spectrum disorders: a longitudinal cohort study. Transl Psychiatry 2015;5:e555.

9. Barch DM, Sheffield JM. Cognitive impairments in psychotic disorders: common mechanisms and measurement. World Psychiatry 2014;13:224-32.

10. Buckholtz JW, Meyer-Lindenberg A. Psychopathology and the human connectome: toward a transdiagnostic model of risk for mental illness. Neuron 2012;74:990-1004.

11. Craddock N, Owen MJ. The Kraepelinian dichotomy-going, going ... but still not gone. Br J Psychiatry 2010;196:92-5

12. Chang EH, Argyelan M, Aggarwal M, et al. The role of myelination in measures of white matter integrity: combination of diffusion tensor imaging and two-photon microscopy of CLARITY intact brains. Neuroimage 2017;147:253-61.

13. Jones DK, Knösche TR, Turner R. White matter integrity, fiber count, and other fallacies: the do's and don'ts of diffusion MRI. Neuroimage 2013;73:239-54.

14. Skudlarski P, Schretlen DJ, Thaker GK, et al. Diffusion tensor imaging white matter endophenotypes in patients with schizophrenia or psychotic bipolar disorder and their relatives. Am J Psychiatry 2013;170:886-98.

15. Jenkins LM, Barba A, Campbell M, et al. Shared white matter alterations across emotional disorders: a voxel-based meta-analysis of fractional anisotropy. Neuroimage Clin 2016;12:1022-34.

16. de Groot JC, de Leeuw F-E, Oudkerk M, et al. Cerebral white matter lesions and depressive symptoms in elderly adults. Arch Gen Psychiatry 2000;57:1071-6.

17. Fields RD. White matter in learning, cognition and psychiatric disorders. Trends Neurosci 2008;31:361-70.

18. Kato T. Molecular genetics of bipolar disorder and depression. Psychiatry Clin Neurosci 2007:61:3-19.

19. Wen Z, Chen J, Khan RAW, et al. Genetic association between NRG1 and schizophrenia, major depressive disorder, bipolar disorder in Han Chinese population. Am J Med Genet B Neuropsychiatr Genet 2016;171B:468-78.

20. Savitz JB, Drevets WC. Imaging phenotypes of major depressive disorder: genetic correlates. Neuroscience 2009;164:300-30

21. Mostaid MS, Mancuso SG, Liu C, et al. Meta-analysis reveals associations between genetic variation in the $5^{\prime}$ and 3 ' regions of neuregulin-1 and schizophrenia. Transl Psychiatry 2017;7:e1004.

22. Stefansson H, Petursson H, Sigurdsson E, et al. Neuregulin 1 and susceptibility to schizophrenia. Am J Hum Genet 2002;71:877-92.

23. Green EK, Raybould R, Macgregor S, et al. Operation of the schizophrenia susceptibility gene, neuregulin 1, across traditional diagnostic boundaries to increase risk for bipolar disorder. Arch Gen Psychiatry 2005;62:642-8.

24. Thomson PA, Christoforou A, Morris S, et al. Association of neuregulin 1 with schizophrenia and bipolar disorder in a second cohort from the Scottish population. Mol Psychiatry 2007;12:94-104.
25. Mostaid MS, Lloyd D, Liberg B, et al. Neuregulin-1 and schizophrenia in the genome-wide association study era. Neurosci Biobehav Rev 2016;68:387-409.

26. Mostaid MS, Lee TT, Chana G, et al. Elevated peripheral expression of neuregulin-1 (NRG1) mRNA isoforms in clozapine-treated schizophrenia patients. Transl Psychiatry 2017;7:1280.

27. McIntosh AM, Hall J, Lymer GKS, et al. Genetic risk for white matter abnormalities in bipolar disorder. Int Rev Psychiatry 2009;21:387-93.

28. Corfas G, Roy K, Buxbaum J. Neuregulin 1-erbB signaling and the molecular/cellular basis of schizophrenia. Nat Neurosci 2004;7: 575-80.

29. Mei L, Xiong WC. Neuregulin 1 in neural development, synaptic plasticity and schizophrenia. Nat Rev Neurosci 2008;9:437-52.

30. Pankonin MS, Sohi J, Kamholz J, et al. Differential distribution of neuregulin in human brain and spinal fluid. Brain Res 2009; 1258:1-11.

31. Mcintosh AM, Moorhead TW, Job D, et al. The effects of a neuregulin 1 variant on white matter density and integrity. Mol Psychiatry 2008;13:1054

32. Wang F, Jiang T, Sun Z, et al. Neuregulin 1 genetic variation and anterior cingulum integrity in patients with schizophrenia and healthy controls. J Psychiatry Neurosci 2009;34:181-6.

33. Bousman CA, Cropley V, Klauser P, et al. Neuregulin-1 (NRG1) polymorphisms linked with psychosis transition are associated with enlarged lateral ventricles and white matter disruption in schizophrenia. Psychol Med 2018;48:801-9.

34. Kastin AJ, Akerstrom V, Pan W. Neuregulin-1-beta1 enters brain and spinal cord by receptor-mediated transport. J Neurochem 2004:88:965-70.

35. Cannon DM, Walshe M, Dempster E, et al. The association of white matter volume in psychotic disorders with genotypic variation in NRG1, MOG and CNP: a voxel-based analysis in affected individuals and their unaffected relatives. Transl Psychiatry 2012;2:e167.

36. Lobbestael J, Leurgans M, Arntz A. Inter-rater reliability of the Structured Clinical Interview for DSM-IV Axis I Disorders (SCID I) and Axis II Disorders (SCID II). Clin Psychol Psychother 2011;18:75-9.

37. Kaufman J, Birmaher B, Brent D, et al. Schedule for Affective Disorders and Schizophrenia for School-Age Children-Present and Lifetime Version (K-SADS-PL): initial reliability and validity data. J Am Acad Child Adolesc Psychiatry 1997;36:980-8.

38. Cui Z, Zhong S, Xu P, et al. PANDA: a pipeline toolbox for analyzing brain diffusion images. Front Hum Neurosci 2013;7:42.

39. Virani K, Jesso S, Kertesz A, et al. Functional neural correlates of emotional expression processing deficits in behavioural variant frontotemporal dementia. J Psychiatry Neurosci 2013;38:174-82.

40. $\mathrm{Wu} \mathrm{O}, \mathrm{Hu} \mathrm{H}, \mathrm{Chen} \mathrm{W}$, et al. Morphological and microstructural brain changes in thyroid-associated ophthalmopathy: a combined voxel-based morphometry and diffusion tensor imaging study. J Endocrinol Invest 2020;43:1591-8.

41. Jiang Q, Chen S, Hu C, et al. Neuregulin-1 (Nrg1) signaling has a preventive role and is altered in the frontal cortex under the pathological conditions of Alzheimer's disease. Mol Med Rep 2016; 14:2614-24.

42. Barnes A, Isohanni M, Barnett JH, et al. Neuregulin-1 genotype is associated with structural differences in the normal human brain. Neuroimage 2012;59:2057-61.

43. Ma N, Li L, Shu N, et al. White matter abnormalities in first-episode, treatment-naive young adults with major depressive disorder. Am J Psychiatry 2007;164:823-6.

44. Wang R, Wang Y, Hu R, et al. Decreased plasma levels of neureglin-1 in drug naive patients and chronic patients with schizophrenia. Neurosci Lett 2015;606:220-4.

45. Shibuya M, Komi E, Wang R, et al. Measurement and comparison of serum neuregulin 1 immunoreactivity in control subjects and patients with schizophrenia: an influence of its genetic polymorphism. I Neural Transm (Vienna) 2010;117:887-95.

46. Corfas G, Roy K, Buxbaum JD. Neuregulin 1-erbB signaling and the molecular/cellular basis of schizophrenia. Nat Neurosci 2004;7:575.

47. Silberberg G, Darvasi A, Pinkas-Kramarski R, et al. The involvement of ErbB4 with schizophrenia: association and expression studies. Am J Med Genet B Neuropsychiatr Genet 2006;141B:142-8.

48. Roy K, Murtie JC, El-Khodor BF, et al. Loss of erbB signaling in oligodendrocytes alters myelin and dopaminergic function, a potential 
mechanism for neuropsychiatric disorders. Proc Natl Acad Sci U S A 2007;104:8131-6

49. Hashimoto R, Straub R, Weickert C, et al. Expression analysis of neuregulin-1 in the dorsolateral prefrontal cortex in schizophrenia. Mol Psychiatry 2004;9:299-307.

50. Hakak Y, Walker JR, Li C, et al. Genome-wide expression analysis reveals dysregulation of myelination-related genes in chronic schizophrenia. Proc Natl Acad Sci U S A 2001;98:4746-51.

51. Bertram I, Bernstein HG, Lendeckel U, et al. Immunohistochemical evidence for impaired neuregulin-1 signaling in the prefrontal cortex in schizophrenia and in unipolar depression. Ann N Y Acad Sci 2007;1096:147-56.

52. Mostaid MS, Lee TT, Chana G, et al. Peripheral transcription of NRG-ErbB pathway genes are upregulated in treatment-resistant schizophrenia. Front Psychiatry 2017;8:225.

53. Kremer D, Göttle P, Hartung H-P, et al. Pushing forward: remyelination as the new frontier in CNS diseases. Trends Neurosci 2016;39:246-63.

54. Lundgaard I, Luzhynskaya A, Stockley JH, et al. Neuregulin and BDNF induce a switch to NMDA receptor-dependent myelination by oligodendrocytes. PLoS Biol 2013;11:e1001743-e.

55. Gunning-Dixon FM, Raz N. The cognitive correlates of white matter abnormalities in normal aging: a quantitative review. Neuropsychology 2000;14:224-32.

56. Thomas AG, Koumellis P, Dineen RA. The fornix in health and disease: an imaging review. Radiographics 2011;31:1107-21.

57. Nestor PG, Kubicki M, Kuroki N, et al. Episodic memory and neuroimaging of hippocampus and fornix in chronic schizophrenia. Psychiatry Res 2007;155:21-8.

58. Paul LK, Lautzenhiser A, Brown WS, et al. Emotional arousal in agenesis of the corpus callosum. Int J Psychophysiol 2006;61:47-56.

59. Wang F, Kalmar JH, Edmiston E, et al. Abnormal corpus callosum integrity in bipolar disorder: a diffusion tensor imaging study. Biol Psychiatry 2008;64:730-3.
60. Metzger CD, Eckert U, Steiner J, et al. High field fMRI reveals thalamocortical integration of segregated cognitive and emotional processing in mediodorsal and intralaminar thalamic nuclei. Front Neuroanat 2010;4:138.

61. Catani M, Dell'Acqua F, De Schotten MT. A revised limbic system model for memory, emotion and behaviour. Neurosci Biobehav Rev 2013;37:1724-37.

62. Camchong J, Lim KO, Sponheim SR, et al. Frontal white matter integrity as an endophenotype for schizophrenia: diffusion tensor imaging in monozygotic twins and patients' nonpsychotic relatives. Front Hum Neurosci 2009;3:35.

63. Emsell L, Leemans A, Langan C, et al. Limbic and callosal white matter changes in euthymic bipolar I disorder: an advanced diffusion magnetic resonance imaging tractography study. Biol Psychiatry 2013;73:194-201.

64. Fitzsimmons J, Kubicki M, Shenton ME. Review of functional and anatomical brain connectivity findings in schizophrenia. Curr Opin Psychiatry 2013;26:172-87.

65. Wang F, Kalmar JH, He Y, et al. Functional and structural connectivity between the perigenual anterior cingulate and amygdala in bipolar disorder. Biol Psychiatry 2009;66:516-21.

66. Drevets WC, Price JL, Furey MLJBs, et al. Brain structural and functional abnormalities in mood disorders: implications for neurocircuitry models of depression. Brain Struct Funct 2008; 213:93-118.

67. Kanaan R, Barker G, Brammer M, et al. White matter microstructure in schizophrenia: effects of disorder, duration and medication. Br J Psychiatry 2009;194:236-42.

68. Mostaid MS, Lee TT, Chana G, et al. Elevated peripheral expression of neuregulin-1 (NRG1) mRNA isoforms in clozapine-treated schizophrenia patients. Transl Psychiatry 2017;7:1280.

69. Harris LW, Pietsch S, Cheng TM, et al. Comparison of peripheral and central schizophrenia biomarker profiles. PLoS One 2012;7:e46368 